\title{
New technologies and research trends for smartphone sensing in intelligent multimedia systems
}

\author{
Seungmin Rho $\cdot$ Wenny Rahayu $\cdot$ Uyen Trang Nguyen
}

Published online: 10 August 2014

(C) Springer-Verlag Berlin Heidelberg 2014

\begin{abstract}
Research progresses in smartphone sensing have been recently explored and developed by various researchers. This special issue on new technologies and research trends for smartphone sensing in intelligent multimedia systems, provides high-quality contributions addressing related, theoretical and practical aspects of intelligent smartphone sensing technologies and their applications. We have selected nine research papers whose topics are strongly related to this special issue.
\end{abstract}

\section{Introduction}

In recent years, one of the world's largest IT market issues is the smartphone. The smartphone has shown its versatility in connecting to the wireless internet or using applications and continues to increase its influence on daily life and business. At first, the cellular phone was a simple device for the voice call, and later, text message exchange. Currently, it has become ubiquitous, and has expanded its functionality to support various types of multimedia applications. Most smartphones are equipped

\footnotetext{
S. Rho $(\square)$

Department of Multimedia, Sungkyul University,

Anyang-si, Korea

e-mail: smrho@sungkyul.edu

W. Rahayu

Department of Computer Science and Computer Engineering,

La Trobe University, Melbourne, Australia

e-mail: W.Rahayu@latrobe.edu.au

U. T. Nguyen

Department of Computer Science and Engineering,

York University, Toronto, Canada

e-mail: utn@cse.yorku.ca
}

with diverse technologies such as cameras, speakers, microphone, and sensors, including GPS, Wi-Fi, accelerometer, Bluetooth, light, and gyro. Sensor data can be used for various purposes such as recognizing user location or inferring user's activity and social context. Using the interactive user interface and sensor devices, it is possible to improve user convenience dramatically and to develop context-aware applications. So far, many studies have been done to pursue these purposes. Therefore, we are looking for efficient and effective, context-sensing algorithms, frameworks, and systems particularly in intelligent multimedia systems.

Each manuscript was blindly reviewed by at least three reviewers consisting of guest editors and external reviewers. After two review processes, nine manuscripts were finally selected to be included in this special issue.

In Sect. 2, we briefly summarize these manuscripts. Section 3 presents a conclusion to this special issue and acknowledgment to the authors and reviewers.

\section{Paper descriptions}

The first paper "Cooperative spectrum sensing via relayassisted random broadcast in cognitive smartphone network" by Zhao et al. [1] proposes a relay-assisted random broadcast scheme to extend the range of message sharing for improving the performance of cooperative spectrum sensing (CSS) by providing a practical power-limited, cluster-based cognitive smartphone network. They also discuss the sensing-sharing tradeoff when their proposed scheme is employed for CSS and show that both the minimum average probability of false alarm and the maximum average probability of detection are unique within the range of the given total sensing time. 
The paper entitled "Triangular inequality-based rotation-invariant boundary image matching for smart devices" by Moon and Loh [2] proposes a reversible data-hiding algorithm that solves the underflow and overflow problems using the location map and embeds more data than other lossless, data-hiding algorithms employing multi-level data hiding with the characteristics of difference image. It is a good solution for sensitive imagery applications, such as military and medical images.

Another paper entitled "Envelope-based boundary image matching for smart devices under arbitrary rotations" by Loh et al. [3] proposes the novel rotationinvariant matching algorithms to significantly reduce the number of distance computations based on the triangular inequality. A new notion of 'self rotation' distance is presented, and it is proven that the self-rotation distance with the triangular inequality produces a tight lower bound and prunes many unnecessary distance computations. A triangular inequality-based algorithm for rotation-invariant image matching is proposed. Then, the self-rotation distance is generalized to define the $k$-self rotation distance, which produces a tighter lower bound and prunes more unnecessary distance computations. An advanced rotationinvariant image matching algorithm based on the $k$-self rotation distance is proposed. It is shown through a series of experiments that the proposed algorithms significantly outperform the existing ones by up to one or two orders of magnitude.

The fourth paper entitled "Robust anonymous authentication protocol for health-care applications using wireless medical sensor networks" by $\mathrm{He}$ et al. [4] proposes an efficient authentication protocol for healthcare applications using WMSNs and claimed their protocol could withstand various attacks. However, authors find that their protocol is vulnerable to the off-line password guessing attack and privileged insider attack. The authors also point out that their protocol cannot provide anonymity. In this paper, authors proposed a robust anonymous authentication protocol for healthcare applications using WMSNs. Compared with [5], the proposed protocol has strong security and computational efficiency. Therefore, it is more suitable for healthcare applications using WMSNs.

The next paper entitled "Real-time smartphone sensing and recommendations towards context-awareness shopping" by Chen et al. [6] proposes a shopping model and implements the smart shopping environment in which the system provides the most appropriate products to customers according to their history of purchasing records by combining customers' personal information and purchasing frequency with customer lifetime value.

The sixth paper entitled "TwitterTrends: a spatio-temporal trend detection and related keywords recommendation scheme" by Kim et al. [7] investigates a spatio- temporal trend detection and related keyword recommendation scheme, which can identify hot keywords and recommend their related keywords at a given location and time by analyzing user tweets and their GPS metadata.

The next paper entitled "The power of smartphones" by Xia et al. [8] describes the typical smartphone computing systems, energy consumption of smartphone, and state-of-theart techniques of energy saving for smartphones. They also propose a location-assisted Wi-Fi discovery scheme, which discovers the nearest Wi-Fi network access points (APs) using the user's location information which allows the user to switch to the Wi-Fi interface in an intelligent manner when the user arrives at the nearest Wi-Fi network AP.

The eighth paper entitled "Smartphone intelligent applications: a brief review" by Rashvand and Hsiao [9] discusses the main features at device level, system level, and application level in the deployment-oriented, objective review of smartphones (SFs). In order to perform a wide range of new and intelligent applications, they propose three classes of optimum solutions: (1) info-smartphones (SF-G0), (2) gadget-smartphones (SF-G1), and (3) professional-smartphones (SF-G2).

In the last paper entitled "User-centric incremental learning model of dynamic personal identification for mobile devices" by Tsai et al. [10] developed the system which focuses on multiview personal identification as captured images vary frequently in the mobile devices. The multiview personal identification is based on body direction detection and incremental learning. The former can provide different new training samples of the same person for the system, whereas the latter is developed to decide whether or not a recognized result should be added to the model. To dynamically select new samples from captured images, candidate analysis and output selection strategy (OSS) are applied to the system. The candidate analysis uses the recognition results to compute vital parameters, which are subsequently delivered to the OSS. The OSS method decides what important information can be learnt. By using these two mechanisms, the robustness of the model is enhanced. Besides, the learning model can adapt itself when different facial views are available. Such a work demonstrates a good example in the mobile environments.

\section{Conclusion}

Finally, our special thanks go to Prof. Thomas Peter Plagemann and all editorial staff for their valuable support throughout the preparation and publication of this special issue. We would like to thank all authors for their contributions to this special issue. We also extend our thanks to the external reviewers for their time and effort in reviewing the manuscripts. 


\section{References}

1. Zhao, Q., Wu, Z., Zhang, D., Shim, M., Yin, C.: Cooperative spectrum sensing via relay-assisted random broadcast in cognitive smartphone network. Multimed. Syst. Springer (2014). doi:10.1007/s00530-014-0385-x. ISSN: 0942-4962

2. Moon, Y.S., Loh, W.K.: Triangular inequality-based rotationinvariant boundary image matching for smart devices. Multimed. Syst. Springer (2014). doi:10.1007/s00530-014-0380-2. ISSN: 0942-4962

3. Loh, W.K., Kim, S.P., Hong, S.K., Moon, Y.S.: Envelope-based boundary image matching for smart devices under arbitrary rotations. Multimed. Syst. Springer (2014). doi:10.1007/s00530014-0386-9. ISSN: 0942-4962

4. He, D., Kumar, N., Chen, J., Lee, C.C., Chilamkurti, N., Yeo, S.S.: Robust anonymous authentication protocol for health-care applications using wireless medical sensor networks. Multimed. Syst. Springer (2013). doi:10.1007/s00530-013-0346-9. ISSN: 0942-4962

5. Kumar, P., Lee, S., Lee, H.: E-SAP: efficient-strong authentication protocol for healthcare applications using wireless medical sensor networks. Sensors 12, 1625-1647 (2012)

6. Chen, C.C., Huang, T.C., Park, J.J., Yen, N.Y.: Real-time smartphone sensing and recommendations towards contextawareness shopping. Multimed. Syst. Springer (2013). doi:10. 1007/s00530-013-0348-7. ISSN: 0942-4962

7. Kim, D., Kim, D., Hwang, E., Rho, S.: TwitterTrends: a spatiotemporal trend detection and related keywords recommendation scheme. Multimed. Syst. Springer (2013). doi:10.1007/s00530013-0342-0. ISSN: 0942-4962

8. Xia, F., Hsu, C.H., Liu, X., Liu, H., Ding, F., Zhang, W.: The power of smartphones. Multimed. Syst. Springer (2013). doi:10. 1007/s00530-013-0337-x. ISSN: 0942-4962

9. Rashvand, H.F., Hsiao, K.F.: Smartphone intelligent applications: a brief review. Multimed. Syst. Springer (2013). doi:10.1007/ s00530-013-0335-z. ISSN: 0942-4962

10. Tsai, H.C., Chen, B.W., Bharanitharan, K., Paul, A., Wang, J.F., Tai, H.C.: User-centric incremental learning model of dynamic personal identification for mobile devices. Multimed. Syst. Springer (2013). doi:10.1007/s00530-013-0328-y. ISSN: $0942-4962$

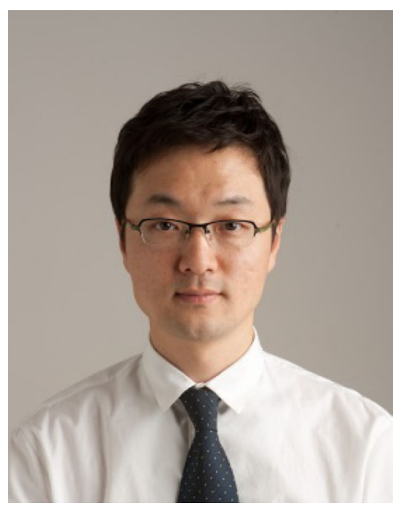

Seungmin Rho received his M.S. and Ph.D. degrees in Computer Science from Ajou University, Korea, in 2003 and 2008, respectively. In 2008-2009, he was a postdoctoral research fellow at the Computer Music Lab of the School of Computer Science in Carnegie Mellon University. In 2009-2011, he had been working as a research professor at School of Electrical Engineering in Korea University. In 2012, he was an assistant professor at Division of Information and Communication in Baekseok University. Dr. Rho is currently a faculty of Department of Multimedia at Sungkyul University. His current research interests include database, big data analysis, music retrieval, multimedia systems, machine learning, knowledge management as well as computational intelligence. He has published 100 papers in refereed journals and conference proceedings in these areas. He has been involved in more than 20 conferences and workshops as various chairs and more than 30 conferences/workshops as a program committee member. He has edited a number of international journal special issues as a guest editor, such as Multimedia Systems, Information Fusion, Engineering Applications of Artificial Intelligence, New Review of Hypermedia and Multimedia, Multimedia Tools and Applications, Personal and Ubiquitous Computing, Telecommunication Systems, Ad Hoc \& Sensor Wireless Networks, etc. He has received a few awards including Who's Who in America, Who's Who in Science and Engineering, and Who's Who in the World in 2007 and 2008, respectively.

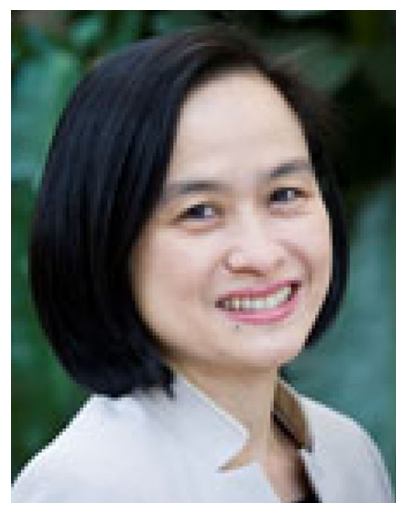

Wenny Rahayu is currently the Head of the Department of Computer Science and Computer Engineering, La Trobe University. Her Ph.D. thesis in the area of Object-Relational Databases has been awarded the best Ph.D. thesis, 2001, by the Computer Science Association Australia (CORE). The main focus of her research is the integration and consolidation of heterogeneous data and systems to support a collaborative environment within a highly datarich environment. To date, she has been the principal investigator or one of the chief investigators of two ARC Linkages, large industry collaboration grants, international grants (Japan JSPS and Australia Indonesia AIGRP), international standard bodies such as Open Geospatial Consortium, Victoria Partnership for Advanced Computing, and the Australia Academy of Science. In the last 10 years, she has published two authored books, three edited books and more than 150 research papers in international journals and proceedings. She has supervised to completion 11 Ph.D.'s, around 32 Honours, and 10 Masters students.

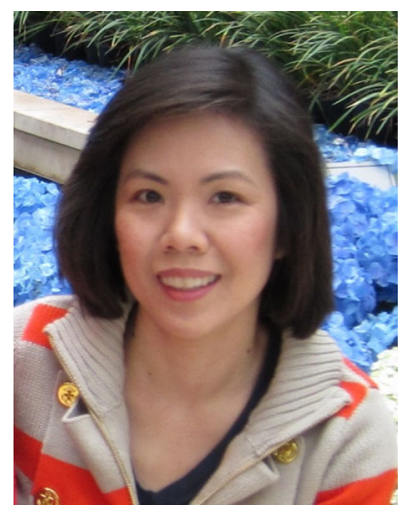

Uyen Trang Nguyen received her Bachelor of Computer Science and Master of Computer Science degrees in 1993 and 1997, respectively, from Concordia University, Montreal, Canada. She completed her Ph.D. degree at the University of Toronto, Canada, in 2003. From 1995 to 1997 she was a software engineer at Nortel Networks, Montreal, Canada. She joined the Department of Computer Science and Engineering at York University,

Toronto, Canada, in 2002 and is currently an associate professor. Her research interests are in the areas of mobile computing, wireless networking, multimedia applications and information security. She is currently the Director of the Graduate Program in Computer Science and Engineering of York University. She was a program co-chair of the 3rd International Conference on Mobile, Ubiquitous, and Intelligent Computing (MUSIC 2012). She was also a program vice-chair of the 13th IEEE International Conference on High Performance Computing and Communications (HPCC 2011) and the 7th International Conference on Future Information Technology (FutureTech 2012). 